$\mathbb{T}$ periodica polytechnica

Mechanical Engineering

$55 / 2(2011) 79,84$

doi: 10.3311/pp.me.2011-2.03

web: http://www.pp.bme.hu/me

(c) Periodica Polytechnica 2011

RESEARCH ARTICLE

\section{Investigation of inner contact and friction conditions of a spherical roller bearing using multi-body simulation}

\author{
Sylvain Fiedler / Timo Kiekbusch / Bernd Sauer
}

Received 2011-11-31

\begin{abstract}
At the Institute of Machine Elements, Gears, and Transmission (MEGT, University of Kaiserslautern) a spherical roller bearing has been modeled in an multi body system (MBS) environment. The use of the commercial MBS (multi-body system) software (MSC ADAMS) allows the development of user-written subroutines for contact recognition and the calculation of contact forces. Those subroutines can help understanding the principles of friction phenomena inside spherical roller bearings, while the measurement of those effects is difficult.Measurements on a friction torque test rig for roller bearings are used to validate the MBS models. Since the sum of all contact forces equals the friction of the bearing, this test stand provides a way for validation of the contact and friction calculations.
\end{abstract}

\section{Keywords}

spherical roller bearing $\cdot$ friction torque $\cdot M B S \cdot$ roller bearing test rig

\section{Acknowledgement}

The authors want to thank the German Research Foundation (Deutsche Forschungsgemeinschaft, DFG) for financial support of this project.

\section{Sylvain Fiedler}

Timo Kiekbusch

Bernd Sauer

Institute of Machine Elements, Gears, and Transmissions (MEGT),University of Kaiserslautern, Kaiserslautern, German

\section{Introduction}

Roller bearings are a commonly used machine elements. Static calculations are often adequate for an analysis of load distribution inside roller bearings. Values like tangential forces between races and rolling elements however can only be determined if the relative movement of the bodies is known. While those effects are not easily accessible by physical measurement the dynamic simulation allows an insight view at limited costs. At the Institute of Machine Elements, Gears, and Transmissions multiple MBS models of cylinder roller bearings and ball bearings have been compiled for the investigation of their inner condition and kinematics. This has not been possible for spherical bearings due to the lack of a model accessible to the researcher. On this account a MBS model is being developed with help from the German Research Funding Organisation (DFG). This model will allow getting precise information about the loads inside the bearing for the design engineer. It will also be a tool for research and development to get data that would only be accessible at high costs if determined by experiments on test rigs. By its fully parametrical design the models open up the possibility to investigate the effects of geometry or load case changes, for example on friction and torque.

\section{Roller bearing test stand}

Considering that the sum of all contact forces equals the friction torque of the bearing, the comparison of this measure can give feedback about the accuracy of the simulation. The necessary experiments are performed on a friction torque test rig for bearings at the Department MEGT. It allows application of complex loads onto bearings. While any combination of radial and axial load can be applied, the shaft can simultaneously be tilted vertically and horizontally to emulate non-perfect alignment [1].

The roller bearing test stand shown in Fig. 1 mainly consists of three components: friction module, loading module and tilting module. Shaft diameters can range from $40 \mathrm{~mm}$ to $110 \mathrm{~mm}$, housing diameters can go up to $200 \mathrm{~mm}$. The outer ring of the test bearing is housed in a hydrostatic bearing. Radial force can be realized in direction of gravity and against. It is applied by a controlled hydraulic cylinder mounted on the loading module 
(see Fig. 2p). It allows the application of load free and slipcritical conditions onto the roller bearing. Axial force is applied by a manually operated hydraulic system at the back of the test stand.

The following values are measured by default: radial load, axial load, friction torque, temperature of outer ring, tilt angles, rotational speed of the shaft and of the bearing's cage. The hy-

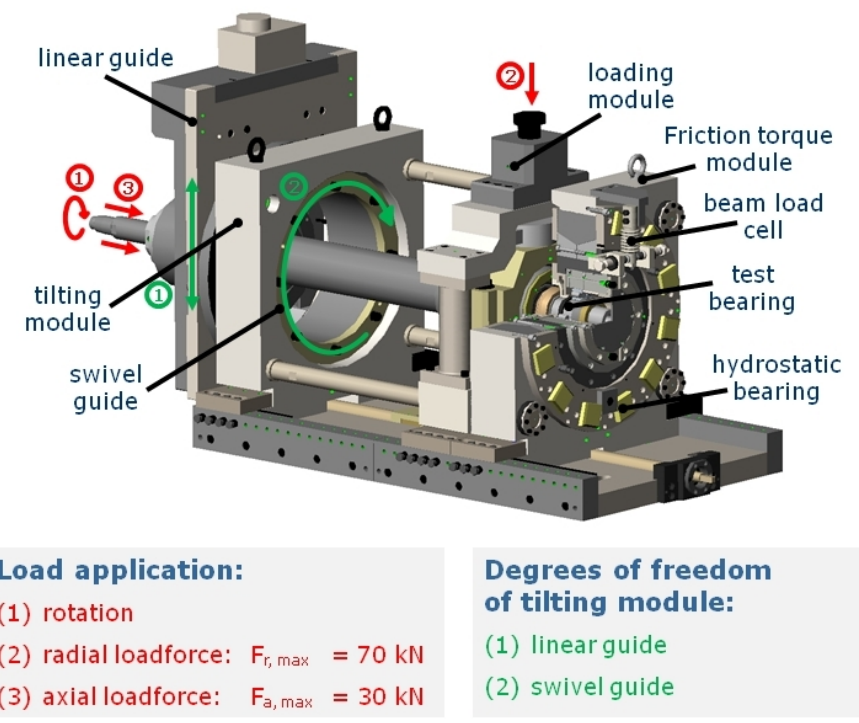

Fig. 1. Roller bearing test stand

drostatic bearing cuts free the rotational degree of freedom. The torque is captured by a load cell, which prevents the hydrostatic bearing from rotating. Both load forces (radial, axial) are measured by load cells. The measuring of the tilting angles is carried out at the shaft's front end by 4 eddy current range sensors shown in Fig. 2 a. The cage slip is detected by an inductive sensor that registers the passing by of the rolling elements.

\section{MBS model}

A fully parametric three-dimensional MBS model of a spherical roller bearing has been developed at the Institute MEGT on the basis of an existing cylinder roller bearing. The main task consisted of elaborating the contact recognition between the rolling elements and the raceways. Other important adaptations were the parametric geometry and the inclination of the rolling bodies.

The cage is modeled in two precisions so far: the virtual cage keeps all rolling elements equidistant and prevents them from tilting. The spring-damper cage allows simulating the cage clearance and the contact stiffness between rolling body and cage. In addition, this cage allows a basic coulomb friction calculation.

To get further insight into the contact characteristics the AST (advanced slicing technique) model has been developed [5] its rotation axis in order to detect edge stress. In contrast to calculations like DIN ISO 281 the slices are not considered independently [6] has an impact on the surrounding ones. The influence intensity is described by weighting factors which de-
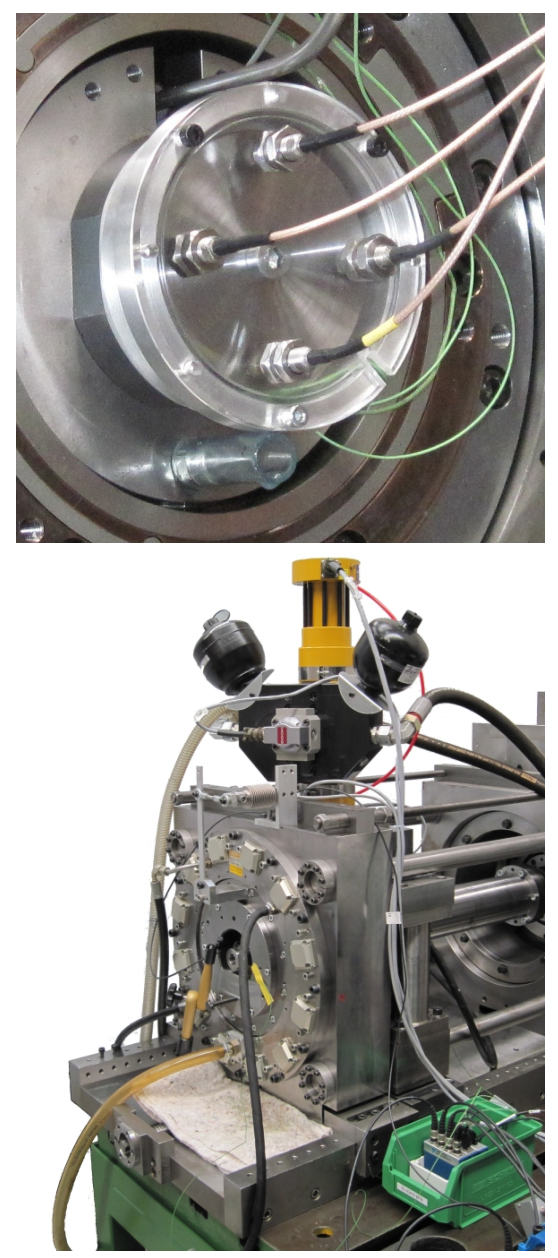

Fig. 2. a) (on the top) measuring the shaft's tilt angles - b) (on the bottom) test stand photo
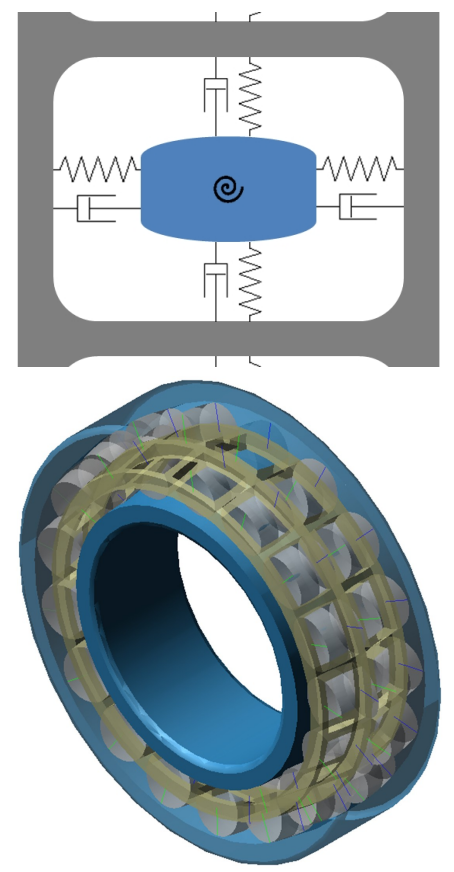

Fig. 3. a) (on the top) spring-damper cage - b) (on the bottom) screenshot of spherical bearing MBS model

crease with raising distance. By discretisation detailed analysis of the friction along the contact surface is permitted. If more detailed information is needed, the discretisation in two dimen- 
sions is possible. Due to high computing times, this level of detail is only applicable for short simulation times [3] times the AST model can be replaced by a conventional model without interdependences of slices.

\section{Friction calculation in the MBS model}

Lubricating oil or grease is commonly used to reduce friction and wear in roller bearings. The generation of a lubricating film in the contact zone is dependent on the conditions of the lubricant, the surface roughness, the load and the current motion between the bodies. The MBS models have diverse friction calculations implemented, they range from parametric approximations to mixed friction with EHD.

\section{Input}

geometry, velocity, material, surface properties, lubricant parameter, normal force, pressures

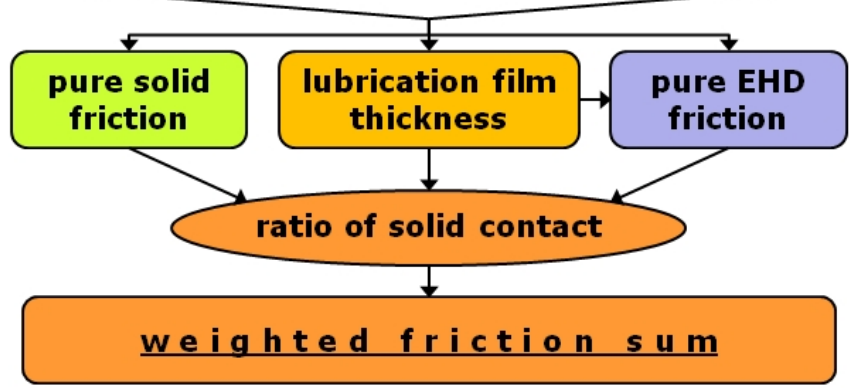

Fig. 4. Method of the MBS friction calculation

To represent mixed friction, solid friction and lubricant friction are calculated separately and summarized by the approach of ZHOU and HOEPRICH [7] dependent of lubricant film height and surface roughness (Fig. 4). Those models were validated by experiments on cylinder roller bearings or ball bearings [1-4].

\section{Validation and simulation results}

Fig. 5] shows the measured and simulated friction torques of a 22212 spherical roller bearing. It has been exposed to radial load up to $20 \mathrm{kN}$. The rise of friction torque over the radial load matches well. The impact of the rotation speed is underestimated by the simulation, in particular at minor loads. This can be explained by the simple contact and friction model integrated into the cage by this time. At higher loads, the MBS model shows an accurate torque prediction which differs only by around 5\%. At small loads, the cage's share in the cumulative friction torque seems to be higher; therefore further development needs to be focused on modeling of cage contacts and friction.

Another validation is the comparison of measured and simulated cage slip. At a constant rotational speed of 3000rpm the spherical bearing was loaded with a slowly and linearly increasing radial force from -600 to $+600 \mathrm{~N}$. At very low forces as it can be seen in Fig. 6 considerable slip occurred (blue diamonds). The simulation (red dots) shows an overestimation of the slip at very low loads, while the qualitative mapping is sat-

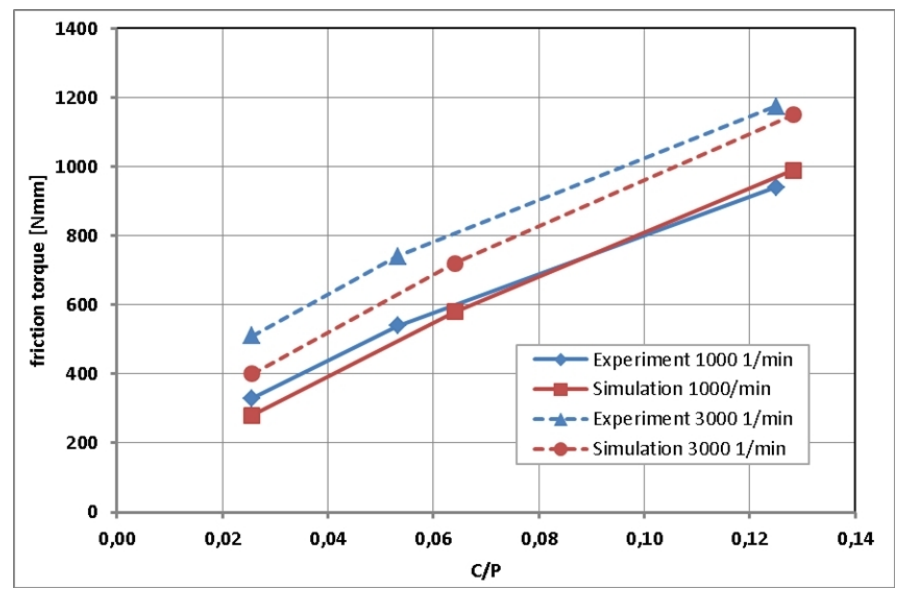

Fig. 5. Comparison of friction torque for validation

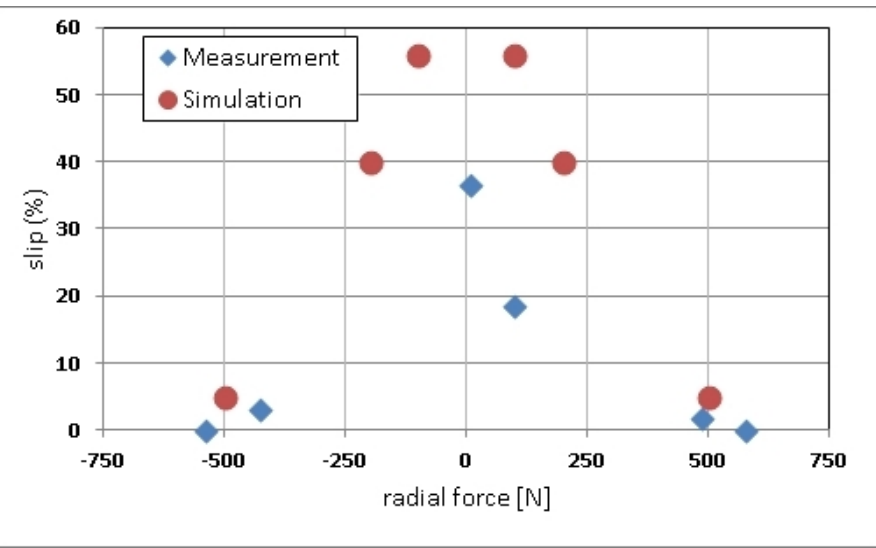

Fig. 6. Comparison of slip for validation

isfactory. This indicates that the development of a cage model based on physical effects should be the next step in development.

The following graphs (Fig. 7. Fig. 10) below show some results obtainable by the MBS simulations concerning the kinematics. The rolling bodies are discretised into 51 slices, the bearing is loaded with $P / C=0.04$ radial load.

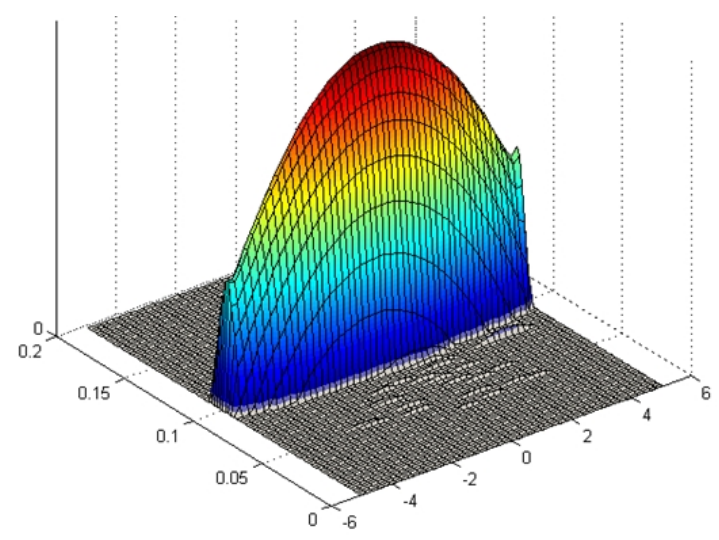

Fig. 7. Pressure distribution plotted over the contact surface

As seen in Fig. 7) on narrow osculation (99\%) the pressure is distributed along the full length of the rolling element even though the load is relatively small. Fig. 8 shows the corresponding distribution of tangential contact forces. The white lines indicate the path where the rolling element has no slip. Tangential forces build up where high pressure comes along with slip. 


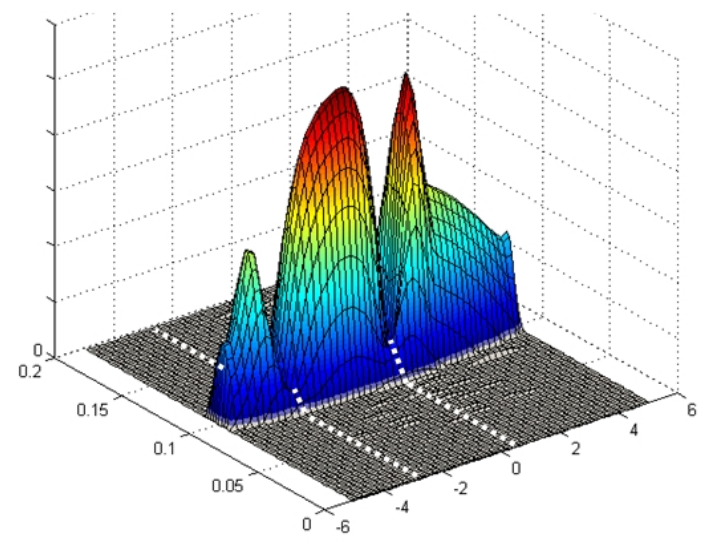

Fig. 8. Distribution of tangential forces along the rolling body axis

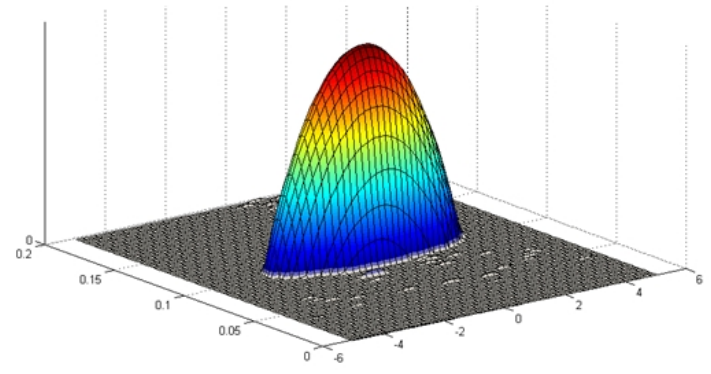

Fig. 9. Pressure distribution on greater osculation

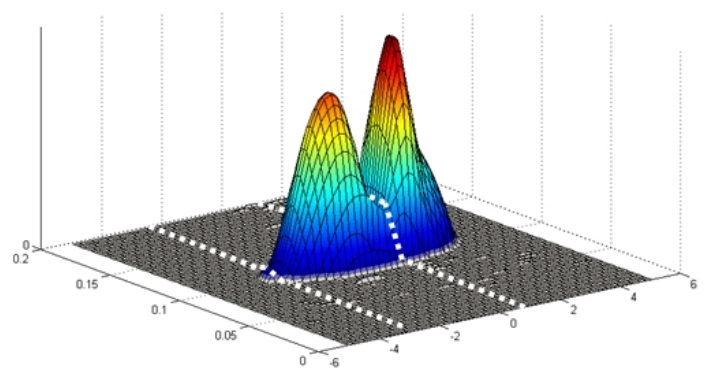

Fig. 10. Distribution of tangential forces on greater osculation

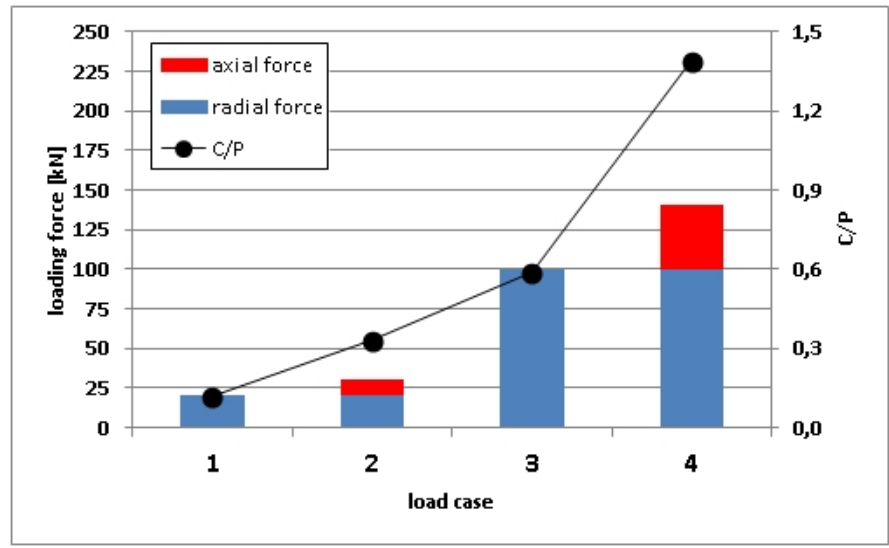

Fig. 11. Simulated load case variations

Fig. 9, Fig. 10 show the same variables at a wider osculation of $95 \%$. The width of the contact area and the tangential forces are reduced.

\section{Variations}

If a MBS model is validated it can be assumed, that the contact description is correct. This means that the model can now be used to investigate different effects or evaluate possible changes in geometry, temperature, lubrication et cetera. In this paragraph, the influence of geometry changes and load changes will be discussed. The four load cases examined are the following (Fig. 11.

$-\operatorname{radial} \operatorname{load} 20 \mathrm{kN}(\stackrel{\wedge}{=} / P=0,12)$

- radial load $20 \mathrm{kN}$ und axial load $10 \mathrm{kN}(\stackrel{\wedge}{=} / P=0,33)$

- radial load $100 \mathrm{kN}(\stackrel{\wedge}{=} / P=0,59)$

- radial load $100 \mathrm{kN}$ und axial load $40 \mathrm{kN}(\stackrel{\wedge}{=} / P=1,39)$

First a reference bearing with standard geometry is loaded with the four different load cases described above. The impact of radial and combined load on the friction torque is seen in Fig. 12 .

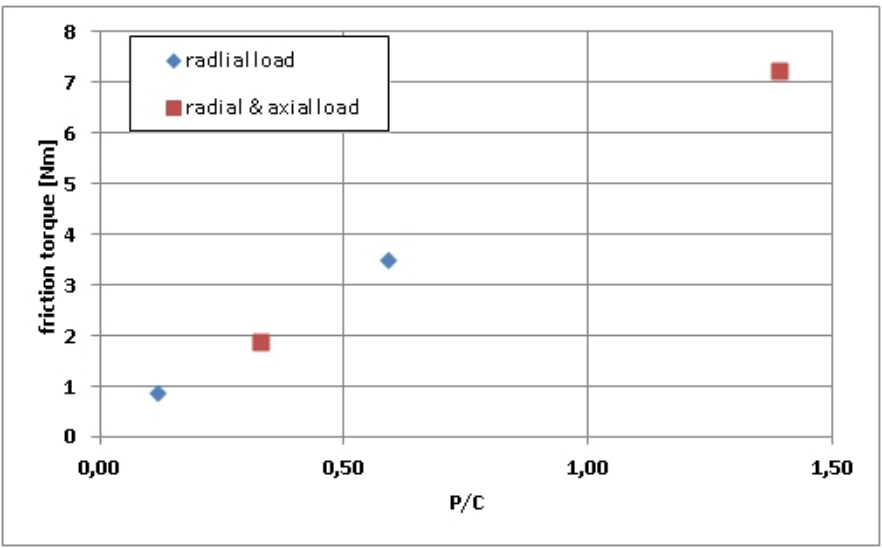

Fig. 12. Friction torque of reference bearing

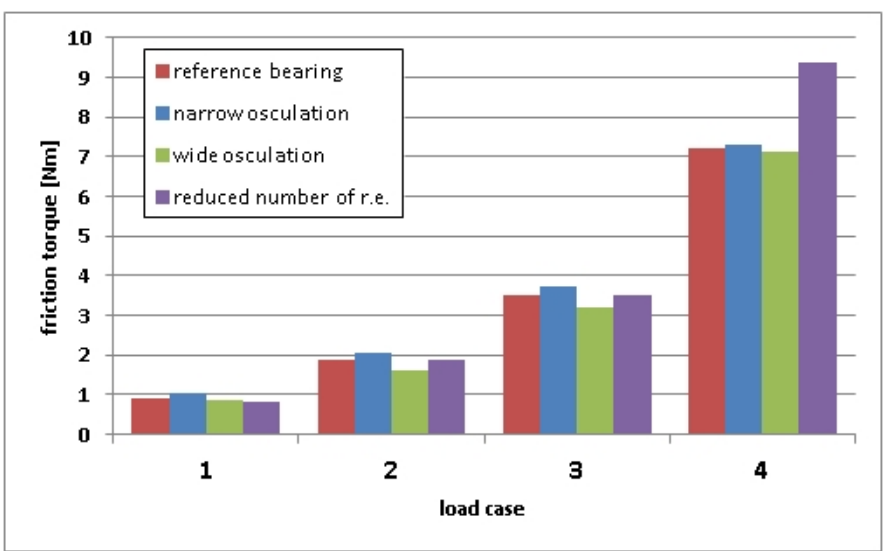

Fig. 13. Friction torque comparison of 4 bearing variations

In order to investigate the influence of geometric changes, these four load cases were also simulated for bearings of the same size, but with changes on internal geometry. Beside the reference bearing, a bearing with narrow osculation has been simulated, a bearing with wide osculation as well as a bearing with less rolling elements (13 instead of 17). The other parameters were kept constant. The rotational speed was $2000 \mathrm{rpm}$ for all simulations. The variations can give a hint about the effects inside the bearing. The results are shown above in Fig. 13 

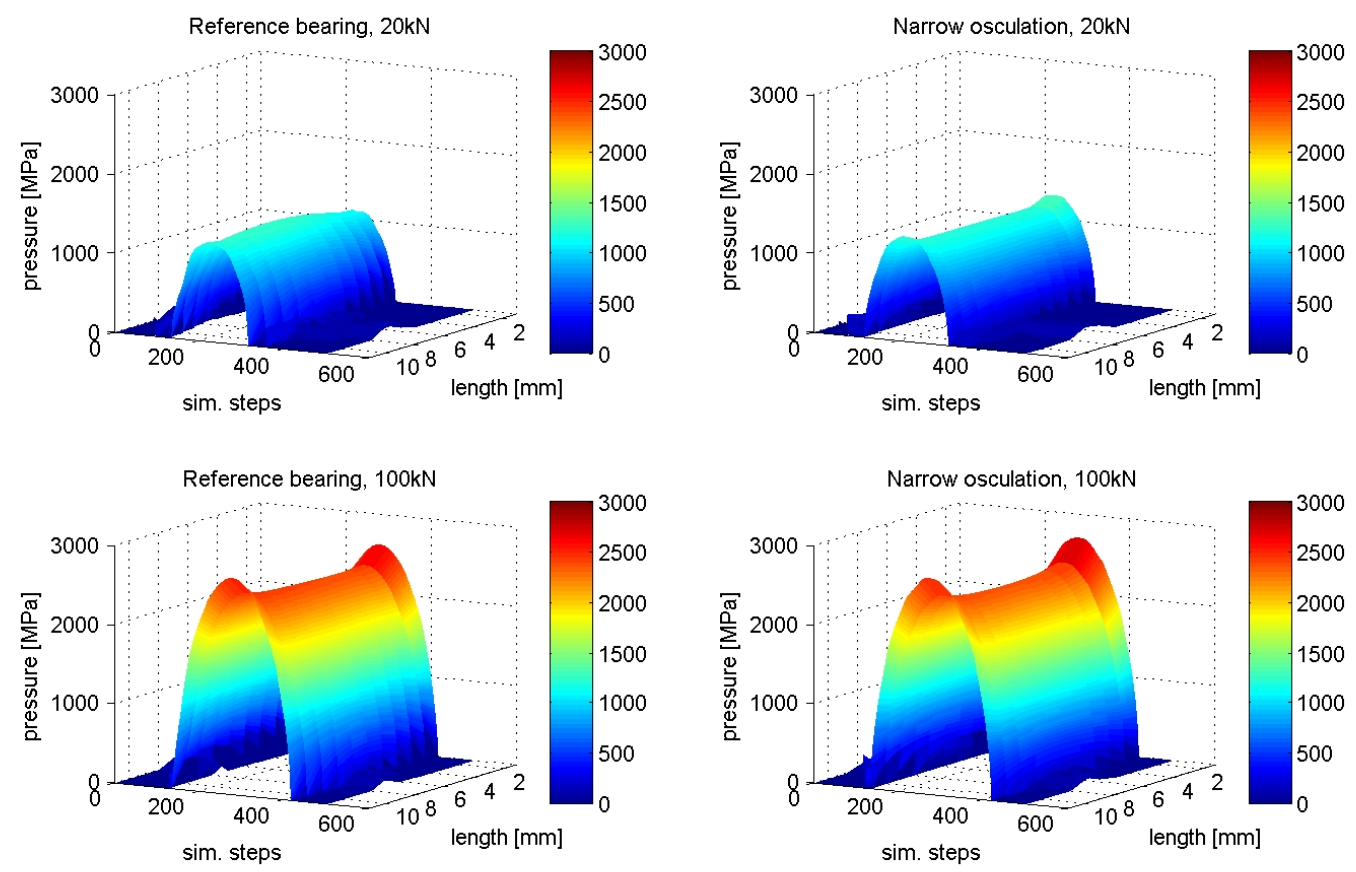

Fig. 14. Pressure distributions for reference bearing and bearing with narrow osculation under radial loads
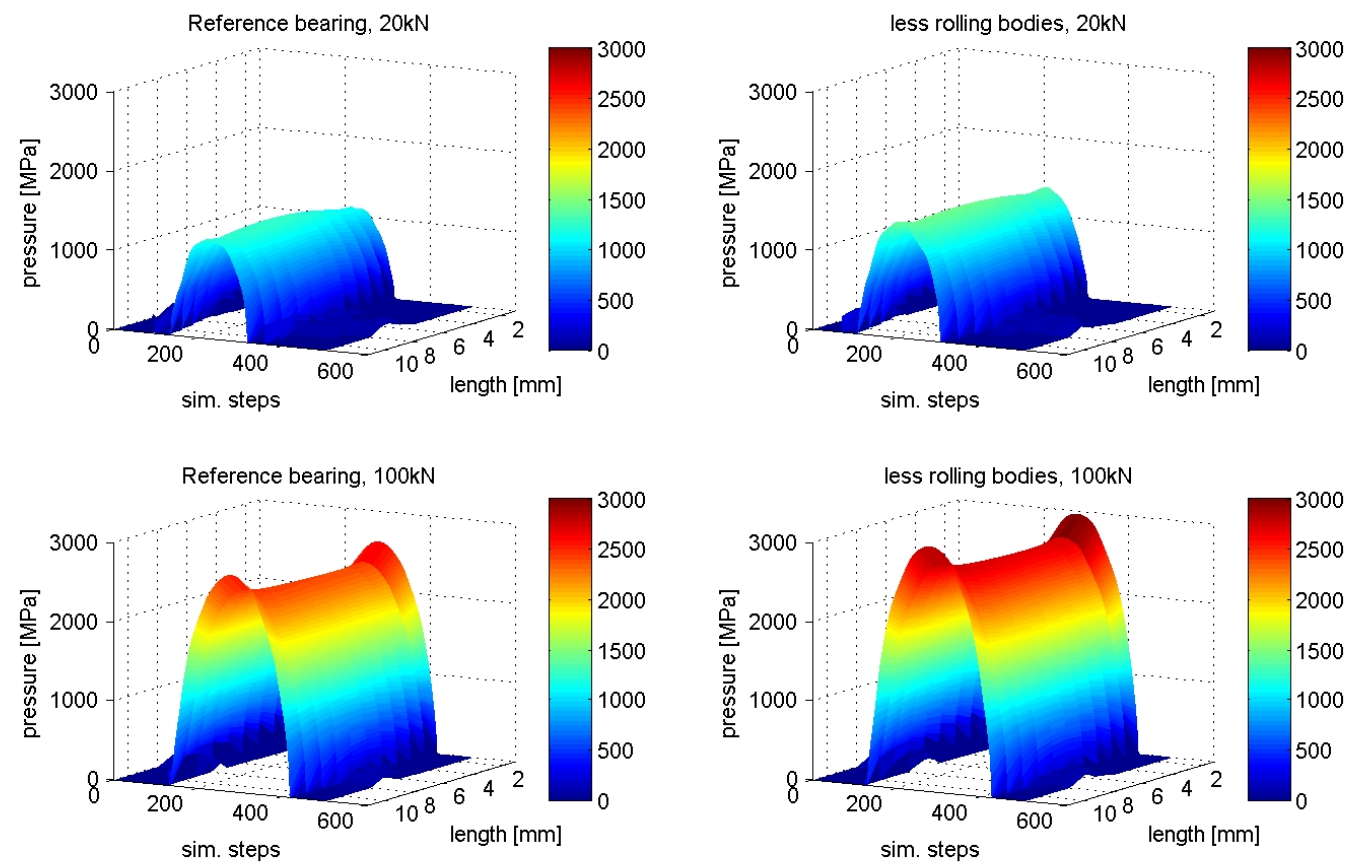

Fig. 15. Pressure distributions for reference bearing and bearing with less rolling elements under radial loads

A narrowing of osculation results in a slightly higher friction torque, especially at low loads, where the contact surface is significantly higher. The difference of the contact surface decreases as loads increase (due to deformation), so the effect of the narrowed osculation decreases. Fig. 14 shows the pressure distributions in the contact. While there is a difference in the pressure distribution for low load (edge stress viewable already for the bearing with narrow osculation), on high loads the pressure distribution looks more similar.
The same effect can be observed by widening the osculation. The friction is slightly lower for low loads, but at very high loads the difference disappears while the magnitude generally rises.

By removal of roller elements the friction decreases slightly. This can be explained by the reduction of contacts, which leads to less friction, even though the contact pressures are higher. At a high load the reduction of rolling bodies generates a torque peak of $130 \%$ compared to the reference bearing. A look at the pressure along the rolling body axis shows directly the elevated 
contact pressure and higher edge stress (7).

\section{Conclusion}

The recently developed MBS model of the spherical roller bearing shows good accuracy for friction torque and cage slip with the experiments. The differences encountered at small loads are the result of the basic cage simulation, which will be the next step of development.

The model presented provides insight into the contact zone and into the kinematics. It can be used to predict friction torque and pressure distribution in dependence of the load case. The results produced can be used to optimize the roller bearing geometry for friction torque reduction and with it the power loss of this machine element. Further investigations can help to get a better understanding of the interaction between bearing, cage, lubricant and loads conditions. The information gained can be used to improve the efficiency of spherical roller bearings in a wide range of operating conditions.

\section{References}

1 Aul E, Sauer B, Analyse von Rillenkugel- und Zylinderrollenlagern unter mehrachsigen Beanspruchungen (Analysis of ball and cylindrical roller bearings under multiaxial loads), VDI-Tagung Gleit- und Wälzlagerungen, VDI Berichte 1942, (2006).

2 Aul V, Kiekbusch T, Marquart M, Sauer B, Experimentelle und simulative Ermittlung von Reibmomenten in Wälzlagern bei Minimalmengenschmierung (Experimental and simulative determination of frictional torque in roller bearings), Tribologie-Fachtagung GfT, 52, (2011). Gesellschaft für Tribologie e.V.

3 Aul V, Marquart M, Kiekbusch T, Fiedler S, Sauer B, WälzlagerDynamikuntersuchung im Pleuel mittels MKS, validiert durch Reibmomente. (Dynamic investigation of roller bearings in connection rods, validated by frictional torque), ATZLive - Reibungsminimierung im Antriebsstrang 2011.

4 Kiekbusch T, Aul E, Marquart M, Sauer B, Experimental and simulative determination of the frictional moment of roller bearings. Industrial and Automotive Lubrication, 18th International Colloquium Tribology, Stuttgart, (2012).

5 Teutsch R, Sauer B, An Alternative Slicing Technique to Consider Pressure Concentrations in Non-Hertzian Line Contacts, Journal of Tribology, 126, (2004), 436-442, DOI 10.1115/1.1739244.

6 Teutsch R, Kontaktmodelle und Strategien zur Simulation von Wälzlagern und Wälzführungen. (Contact models and strategies for simulation of roller bearings), Dissertation. TU Kaiserslautern,Shaker-Verlag, (2005).

7 Zhou RS, Hoeprich MR, Torque of Tapered Roller Bearings, Journal of Tribology, 113, (1991), 590-597, DOI 10.1115/1.2920664. 